\title{
RASGOS DEL ENVEJECIMIENTO DE LA POBLACIÓN EN LA COMUNIDAD VALENCIANA
}

\author{
Fermín Bonmatí Antón \\ Consuelo Sebastiá Llinares
}

\begin{abstract}
RESUMEN
En la Comunidad Valenciana se aprecia una fuerte correlación entre nivel demográfico de los municipios y nivel de envejecimiento, medido este último en función del porcentaje de población con 65 o más años sobre el total del censo municipal. Aquellos municipios con menos de 2.000 hab. ofrecen, por lo general, grados de vejez más acentuados que los que están por encima de dicho nivel demográfico. Por otro lado, se aprecian niveles de envejecimiento mayores en la Montaña alicantina y en el interior de las provincias de Valencia y sobre todo Castellón que en los espacios litoral y el Sur de la Comunidad. Hemos comprobado la confirmación en 1986 de las situaciones que se daban cinco años antes.
\end{abstract}

Palabras clave: Grado de envejecimiento, Comunidad Valenciana, Año 1986, Perspectivas.

\section{RÉSUMÉ}

A la région de Valènce (en Espagne) on estime une forte corrélation entre niveau démographique des communes et niveau de vieillisement, sélon le pourcentage de population avec 65 ou plus agées sur la totalité du recensement de la commune. Les communes avec moins 2.000 hab. montrent, en général, dégrés de vieillissement plus accentués que ceux qui sont par dessus le niveau démographique de reference. D'autre part, on vérifie niveaux de vieillissement plus forts à la Montaña d'Alicante et à l'interieur des provinces de Valence et surtout Castellon, que aux espaces du litoral et au sud de la région. Nous avons constaté la confirmation, à 1986, des situations qui l'on avait vu à 1981.

Mots cléfs: Dégrée de vieillissement, Région de Valènce, Année 1986, Perspectives.

La Comunidad Valenciana queda caracterizada por la dicotomía interior -litoral en los planos demográfico y económico. La oposición entre ambas zonas se manifiesta, entre otras variables, en un grado de envejecimiento elevado en la parte interna de la región, frente a términos municipales de los espacios litoral y prelitoral con niveles de juventud 
acentuados. Sin embargo, la práctica totalidad de la provincia de Castellón quedaría inscrita en el indicado espacio de mayor índice de vejez, al que también se suman las comarcas del Noroeste y Oeste de la provincia de Valencia, y la Montaña alicantina. En contrapartida, la Plana castellonense, las llanuras y el Sur de la provincia de Valencia, y la mayoría del espacio provincial de Alicante, tienen censos más jóvenes. Esta hipótesis quedó demostrada para el año 1981 por el profesor Gozálvez. Según este autor, existen interesantes relaciones entre acusado grado de envejecimiento y municipio rural, e índice de envejecimiento y lejanía o alejamiento de centros urbanos. Asimismo, pudo comprobar que el fenómeno del envejecimiento se extiende por espacios plurimunicipales como un fenómeno de contigüidad, en manchas crecientes. Comarcas como el Alto Mijares, Alto Palancia, Montaña alicantina y en menor medida los Serranos y el Valle de Ayora - Canal de Navarrés, destacan como espacios con altos índices de vejez ${ }^{1}$. Por otra parte, Ismael Vallés ha calculado los índices de vejez de las comarcas valencianas en 1986 -(población con 65 y más años / población con menos de 15 años) x 100-, y ha demostrado igualmente la existencia de dos áreas: a) comarcas con índices bajos, donde la dinámica económica ha incidido atrayendo población -muchas veces procedente del interior de la propia Comunidad Valenciana-, y donde también se dan las tasas de fecundidad más altas de la región; b) espacios comarcales con índices de vejez altos, emisores de población hacia las anteriores y con tasas de fecundidad bajas ${ }^{2}$. Se puede constatar la coincidencia de las primeras con las áreas de menor grado de envejecimiento señaladas en el litoral -sobre todo en l'Hortay Sur de la Comunidad, mientras que en éstas últimas, en el interior, los niveles de vejez son superiores.

Pretendemos ahora estudiar la dinámica seguida por el envejecimiento entre 1981 y 1986, fecha en que se confeccionó el último Padrón de Población en España. Con este propósito y utilizando las series estadísticas editadas por la Conselleria d'Economia i Hisenda, se ha calculado la proporción de individuos con más de 65 años de edad censados en los municipios de la Comunidad Valenciana.

Habida cuenta que en 1981 los grados de envejecimiento más elevados correspondían, en líneas generales, a los municipios con menos de 2.000 hab. de hecho, el estudio se centrará en éstos, efectuando comparaciones con aquéllos otros cuyo censo supere dicho umbral.

\section{Niveles de envejecimiento en la Comunidad Valenciana}

En el cuadro I se sintetiza el proceso de envejecimiento para toda la Comunidad Valenciana y cada una de sus tres provincias, en función de los porcentajes que representa la población de 65 años o más sobre el total de los censos municipales. El promedio de la Comunidad Valenciana es del 11'7 por ciento en 1986, ligeramente por debajo de la media nacional en la misma fecha $(12 ' 2 \%)$.

Utilizando la terminología de P. Paillat, cuando el porcentaje de viejos oscila entre 16 y 19,9 por ciento del censo se considera que se trata de municipios «en transición»; cuando va del 20 al 23'9 por ciento, de municipios «viejos»; y con porcentajes iguales o superiores al 24 por ciento se les califica como municipios «muy viejos» ${ }^{3}$. En este sentido, tanto la Comunidad Valenciana como el conjunto del territorio nacional pueden ser considerados como espacios «jóvenes», por tener porcentajes inferiores al 16 por ciento.

En aras de una mayor simplificación se ha clasificado a los términos de la Comunidad en tres grandes grupos, según los criterios seguidos por el I.N.E. en la elaboración de las 
Cuadro I

COMUNIDAD VALENCIANA. SITUACIONES MUNICIPALES SEGÚN GRADO DE ENVEJECIMIENTO EN LA COMUNIDAD VALENCIANA

\begin{tabular}{|c|c|c|c|c|c|c|c|c|}
\hline \multirow{3}{*}{$\begin{array}{l}\text { ALICANTE } 198 \\
\end{array}$} & \multicolumn{2}{|c|}{ RURALES } & \multicolumn{2}{|c|}{ INTERM. } & \multicolumn{2}{|c|}{ URBANOS } & \multicolumn{2}{|c|}{ TOTAL } \\
\hline & \multirow[t]{2}{*}{ N. ${ }^{\circ}$} & \multirow[t]{2}{*}{ Hab. } & \multirow[t]{2}{*}{ N. ${ }^{\circ}$} & \multirow[t]{2}{*}{ Hab. } & \multirow[t]{2}{*}{ N. ${ }^{\circ}$} & \multirow[t]{2}{*}{ Hab. } & \multirow[t]{2}{*}{$\mathbf{N} .^{\circ}$} & \multirow[t]{2}{*}{ Hab. } \\
\hline & & & & & & & & \\
\hline EN TRANS. & 13 & 12.897 & 3 & 15.631 & - & - & 16 & 28.528 \\
\hline VIEJOS & 15 & 12.649 & 1 & 3.426 & - & - & 16 & 16.075 \\
\hline MUY VIEJO & 29 & 11.719 & - & - & - & - & 29 & 11.719 \\
\hline TOTAL & 57 & 37.265 & 4 & 19.057 & - & - & 61 & 56.322 \\
\hline \multicolumn{9}{|c|}{ ALICANTE 1986} \\
\hline EN TRANS. & 18 & 17.361 & 3 & 12.898 & - & - & 21 & 30.259 \\
\hline VIEJOS & 17 & 7.873 & 1 & 5.237 & - & - & 18 & 13.110 \\
\hline MUY VIEJO & 27 & 11.747 & - & - & - & - & 27 & 11.747 \\
\hline TOTAL & 62 & 36.981 & 4 & 18.135 & - & - & 66 & 55.116 \\
\hline \multicolumn{9}{|c|}{ VALENCIA 1981} \\
\hline EN TRANS. & 29 & 24.836 & 14 & 32.826 & - & - & 43 & 57.662 \\
\hline VIEJOS & 25 & 23.656 & 2 & 4.876 & - & - & 27 & 28.532 \\
\hline MUY VIEJO & 28 & 12.814 & 1 & 2.257 & - & - & 29 & 15.071 \\
\hline TOTAL & 82 & 61.306 & 17 & 39.959 & - & - & 99 & 101.265 \\
\hline \multicolumn{9}{|c|}{ VALENCIA 1986} \\
\hline EN TRANS. & 48 & 40.122 & 6 & 15.096 & - & - & 54 & 55.218 \\
\hline VIEJOS & 20 & 11.326 & 3 & 6.427 & - & - & 23 & 17.753 \\
\hline MUY VIEJO & 27 & 15.630 & - & - & - & - & 27 & 15.630 \\
\hline TOTAL & 95 & 67.078 & 9 & 21.523 & - & - & 104 & 88.601 \\
\hline \multicolumn{9}{|c|}{ CASTELLÓN 1981} \\
\hline EN TRANS. & 14 & 10.780 & 8 & 30.721 & - & - & 22 & 41.501 \\
\hline VIEJOS & 26 & 18.608 & 5 & 13.279 & - & - & 31 & 31.887 \\
\hline MUY VIEJO & 57 & 28.066 & 1 & 2.011 & - & - & 58 & 30.077 \\
\hline TOTAL & 97 & 57.454 & 14 & 46.011 & - & - & 101 & 103.465 \\
\hline \multicolumn{9}{|c|}{ CASTELLÓN 1986} \\
\hline EN TRANS. & 10 & 8.890 & 4 & 14.311 & - & - & 14 & 23.201 \\
\hline VIEJOS & 20 & 18.751 & 5 & 13.761 & - & - & 25 & 32.512 \\
\hline MUY VIEJO & 72 & 29.927 & 1 & 2.121 & - & - & 73 & 32.048 \\
\hline TOTAL & 102 & 57.568 & 10 & 30.193 & - & - & 112 & 87.761 \\
\hline \multicolumn{9}{|c|}{ C. VALENC. 1981} \\
\hline EN TRANS. & 56 & 48.513 & 25 & 79.178 & - & - & 81 & 127.691 \\
\hline VIEJOS & 66 & 54.910 & 8 & 21.581 & - & - & 74 & 76.491 \\
\hline MUY VIEJO & 114 & 52.599 & 2 & 4.268 & - & - & 116 & 56.867 \\
\hline TOTAL & 236 & 156.022 & 35 & 105.027 & - & - & 271 & 261.049 \\
\hline \multicolumn{9}{|c|}{ C. VALENC. 1986} \\
\hline EN TRANS. & 76 & 66.373 & 13 & 42.305 & - & - & 89 & 108.678 \\
\hline VIEJOS & 57 & 37.950 & 9 & 25.425 & - & - & 66 & 63.375 \\
\hline MUY VIEJO & 126 & 57.304 & 1 & 2.121 & - & - & 127 & 59.425 \\
\hline TOTAL & 259 & 161.627 & 23 & 69.851 & - & - & 282 & 231.478 \\
\hline
\end{tabular}


estadísticas: rurales o municipios de menos de 2.000 hab.; intermedios, entre 2.000 y 9.999 hab.; y urbanos, los de 10.000 hab. o más.

Del análisis de dicho cuadro se desprende una evolución contrapuesta entre las variables número de municipios y de habitantes, puesto que mientras el número de municipios en cualquiera de las tres situaciones de envejecimiento es mayor en 1986 que en 1981 para el conjunto de toda la Comunidad Valenciana, desciende en cambio la cifra absoluta de población correspondiente a los mismos municipios.

Por categorías, y siempre refiriéndonos a la región en su conjunto, hay que señalar el incremento del número de municipios y también de habitantes en la de «muy viejos». En cambio, se aprecia un descenso de ambas variables para los municipios «viejos». Por último, es la categoría «en transición» la única que cumple la tendencia general, al aumentar el número de municipios pero descender el de habitantes.

Es muy significativa la ausencia de municipios urbanos con porcentajes de población vieja superiores al 16 por ciento en ambas fechas. El fenómeno del envejecimiento se centra básicamente en los municipios rurales; en esta situación también se encuentran algunos municipios intermedios, aunque con mayoría en situación «en transición».

En efecto, este fenómeno se concentra cada vez más entre los municipios con menos de 2.000 hab., de tal modo que para esta clase de términos se aprecia un aumento tanto del número de municipios como de la cifra de habitantes. En los intermedios, en cambio, se produce en el intercensal un descenso de ambas variables en el conjunto de la Comunidad Valenciana. Ciñéndonos a las categorías de envejecimiento, hay que subrayar igualmente una oposición de tendencias según se trate de municipios rurales o intermedios. Tanto en 1981 como en 1986 tiene mayor peso específico la situación «en transición» (porcentajes de población vieja entre el 16 y el 19 por ciento) entre los intermedios, mientras para los rurales predomina la de «muy viejos» (porcentajes superiores al 24 por ciento).

Prueba de la concentración del fenómeno del envejecimiento entre los municipios rurales, es el aumento entre las dos fechas de referencia del número de municipios y de habitantes en la situación de «muy viejos»; ello deriva de la transformación de municipios que en 1981 estaban considerados como «viejos» o «en transición», mientras en 1986 han alcanzado la situación de «muy viejos». También se produce un incremento del número de municipios «en transición», sobre todo por el paso de algún municipio «joven» en 1981 a dicha situación en 1986. Entre los municipios «viejos», en cambio, se aprecia un menor número de casos y de habitantes, por su conversión en «muy viejos».

Tendencia contraria se observa en la evolución de los municipios intermedios, hacia el rejuvenecimiento. Prueba de ello es el predominio del apartado «en transición» en ambas fechas, y también la existencia de menor número de municipios en 1986 que en 1981, combinando las tres situaciones de envejecimiento.

El porcentaje de población vieja es inversamente proporcional al tamaño medio de los municipios de cada categoría: cuando menor es la media de habitantes mayor es el índice de vejez.

Por provincias, Castellón da el mayor número de municipios envejecidos, 112 sobre un total de 143 en 1986. El tamaño demográfico medio del municipio en situación de envejecimiento es en esta provincia inferior a la cifra promedio de la de Valencia, con lo que ésta última tiene en conjunto un mayor contingente de población envejecida. Valencia cuenta con 104 municipios envejecidos de un total de 263, en 1986. Alicante, con un municipio tipo de mayor censo que en Castellón, da 66 municipios envejecidos sobre $138 \mathrm{y}$, por tanto, menor volumen de población en esta situación. Castellón y Valencia cumplen la tendencia general de toda la Comunidad, por cuanto se da, en el intercensal, un incre- 
mento del número de municipios y un descenso de las cifras de población envejecida. Alicante y Valencia presentan en 1986 menos municipios en la situación de «muy viejos» que en 1981; sin embargo, la población correspondiente apenas acusa la variación. Además, en Alicante, todos los municipios con mayor envejecimiento son de tipo rural. Castellón, en cambio, experimenta un notable incremento de municipios y de habitantes en el apartado «muy viejos».

En la categoría de «viejos» (porcentaje de viejos entre el 20 y el 24 por ciento) sólo Valencia cumple la norma general de toda la Comunidad, es decir, incremento de la cifra de municipios y de habitantes. En Alicante crece el número de municipios entre 1981 y 1986. Castellón, sin embargo, tiene menos términos municipales de este tipo pero el cómputo global de población es superior en la segunda fecha.

El mayor grado de envejecimiento en la provincia de Castellón con respecto a las otras dos, también se manifiesta en la disminución de los municipios y la población en el apartado de municipios «en transición», mientras en Alicante hay más municipios y más habitantes, ratificando el hecho de ser la provincia con menor acentuación del envejecimiento.

En resumen, en Castellón, sobre un envejecimiento ya más acusado en 1981, entre 1981 y 1986 se intensifica dicho fenómeno. Así, en primer lugar, aumenta el número de municipios «muy viejos». Por otro lado, frente a la norma general de toda la Comunidad, hay mayor cantidad de municipios «viejos» que «en transición» en 1986, y también se aprecia un descenso en el número de municipios y de habitantes rurales para la situación «en transición». Asimismo, es la provincia con más municipios en cualquiera de las tres situaciones consideradas, a pesar de tener menos municipios que la provincia de Valencia.

Valencia y Alicante permanecen estables, con una ligera tendencia al «rejuvenecimiento» -siempre dentro del contexto de municipios rurales-, como demuestran el aumento de los municipios y los habitantes en municipios «en transición» entre ambas fechas, y el descenso de municipios «muy viejos», aunque aquí la población se estabilice.

Castellón, a pesar de ser la provincia con mayor descenso en cifras absolutas y

Cuadro II

COMUNIDAD VALENCIANA. POBLACIÓN DE LOS MUNICIPIOS ENVEJECIDOS EN 1981 Y 1986 (LA POBLACIÓN CON 65 AÑOS O MÁS SUMA MÁS DEL 16\% DEL CENSO)

\begin{tabular}{|c|c|c|c|c|c|c|c|c|}
\hline & \multirow[b]{3}{*}{1981} & \multirow[b]{3}{*}{1986} & \multirow{3}{*}{$\begin{array}{c}\text { A } \\
\text { Variación } \\
\text { 1981-86 }\end{array}$} & \multicolumn{5}{|c|}{ B } \\
\hline & & & & \multirow{2}{*}{$\begin{array}{c}\% \text { de } \\
\text { variación }\end{array}$} & \multicolumn{2}{|c|}{ Pobl. total } & \multirow[t]{2}{*}{1} & \multirow[t]{2}{*}{2} \\
\hline & & & & & 1981 & 1986 & & \\
\hline Alicante & 56.322 & 55.116 & -1.516 & $-2,7$ & 1.148 .597 & 1.254 .920 & $4^{\prime} 9$ & $4^{\prime} 4$ \\
\hline Castellón & 103.465 & 87.761 & -15.704 & $-15,2$ & 431.755 & 437.320 & $20^{\prime} 0$ & $20^{\prime} 1$ \\
\hline Valencia & 101.265 & 88.601 & -12.664 & $-12,5$ & 2.066 .413 & 2.079 .762 & $4^{\prime} 9$ & 4'3 \\
\hline C. Valenciana & 261.049 & 231.478 & -29.571 & $-11,3$ & 3.646 .765 & 3.772 .002 & $7^{\prime} 1$ & 6'1 \\
\hline
\end{tabular}

$\mathrm{A}=$ Población total entre las tres situaciones de envejecimiento

$\mathrm{B}=$ Población total de la provincia o región

$1=\%$ de A sobre B en 1981

$2=\%$ de A sobre B en 1986

Fuente: Elaboración propia a partir del Padrón Municipal de Habitantes de 1986 
relativas de la población vieja entre 1981 y 1986, es sin embargo y con mucha diferencia la que tiene mayor grado de envejecimiento, por cuanto el contingente global de población residente en los municipios envejecidos (aquellos cuya población de 65 años y más representan como mínimo el 16 por ciento de sus censos), supone la quinta parte de la totalidad de la población provincial en 1981 y 1986. En Alicante y Valencia, en cambio, este tipo de población no representa siquiera el 5 por ciento en ninguna de las dos fechas.

Estas elevadas cifras relativas de Castellón responden a la existencia de gran número de municipios con menos de 2.000 hab., en la mayor parte de los cuales -y también en algunos que superan estas cifras de referencia- se ha producido en las últimas décadas un acentuado proceso de éxodo rural hacia las cabeceras comarcales y centros urbanos del litoral castellonense. El fenómeno ha estado protagonizado sobre todo por población adulta, mientras que los contingentes de mayor edad permanecen con más frecuencia en los municipios rurales. Estos déficits de población adulta repercuten en el incremento relativo de los índices de vejez.

\section{Reparto espacial de los índices de vejez en 1986}

El envejecimiento no se reparte de forma uniforme en toda la Comunidad Valenciana, pues existen grandes diferencias según el tamaño demográfico de los municipios, y dentro del mismo nivel también hay que señalar diferencias entre unos espacios y otros, en función de factores como: aislamiento respecto a los grandes centros urbanos, disponibilidad de tierras para el cultivo, distribución de terrenos de secano y regadío, red tradicional de poblamiento y factores locales más específicos.

\section{Grados de envejecimiento por comarcas}

En la fig. 1 se representa la situación de envejecimiento de cada una de las comarcas de la Comunidad Valenciana, según los niveles de población joven, adulta y vieja en 1986. A tal efecto, se aprecia que son l'Horta Oest, l'Horta Sud y Baix Vinalopó, los espacios con menor grado de vejez, siempre con una población senil por debajo del 10\%. Por contra, una serie de espacios comarcales en el interior de Castellón y Valencia -los Serranos, els Ports, l'Alt Maestrat, Alto Palancia, Alto Mijares y Rincón de Ademuz- resultan ser los más envejecidos de la Comunidad, pues los jóvenes casi nunca alcanzan el $20 \%$ del total del censo, mientras que los viejos representan entre la quinta y la tercera parte de la población.

Por provincias, la de Alicante no tiene comarcas con alto grado de envejecimiento. Las más envejecidas son el Comtat y la Marina Alta o Marquesat de Dènia, en pleno ámbito montañoso, con promedios de población vieja entre el 15 y el 20 por ciento. El Sur de la provincia, es decir, el Bajo Segura y Baix Vinalopó, se encuentran entre las categorías que muestran menor grado de envejecimiento, siendo la cota superior de población de 65 y más años la del 15\%. Los cursos alto y medio del Vinalopó quedan igualmente catalogados como espacios de bajo índice de vejez, al igual que otras comarcas litorales -l'Alacantí y la Marina Baixa- junto con l'Alcoià, al Norte de la provincia. La presencia de grandes y medianos centros urbanos repartidos por todo el ámbito provincial de Alicante, con un alto grado de industrialización y/o terciarización en la mayor parte de ellos, ha servido para frenar el éxodo rural y en ocasiones para convertirse en foco de atracción de población inmigrante extraprovincial. Las comarcas con más población 


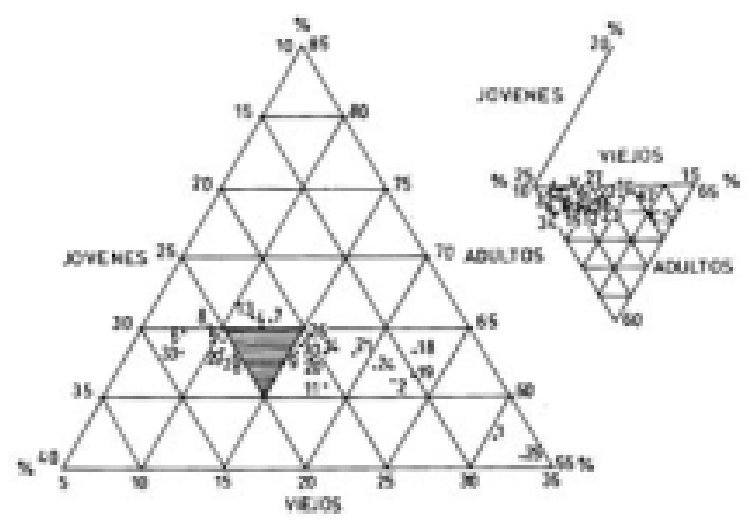

1. Rincón de Ademuz

2. Los Serranos

3. Camp de Turia

4. Camp de Morvedre

5. Horta N.

6. Horta O.

7. Valencia capital

8. Horta S.

9. Plana de Requena y Utiel

10. Hoya de Buñol

11. Valle de Ayora

12. Ribera Alta

13. Ribera Baja

14. Canal de Navarrés

15. La Costera

16. Vall d'Albaida

17. La Safor

18. Els Ports de Morella

19. Alt Maestrat

20. Baix Maestrat
21. L'Alcalatén

22. Plana Alta

23. Plana Baja

24. Alto Palancia

25. Alto Mijares

26. El Comtat

27. L'Alcoià

28. Alto Vinalopó

29. Vinalopó Medio

30. Marina Alta

31. Marina Baja

32. L'Alacantí

33. Bajo Vinalopó

34. Bajo Segura

A. ALICANTE (provincia)

CS. CASTELLÓN (provincia)

$\mathrm{V}$. VALENCIA (provincia)

FIGURA 1. Estructura por edades (diagrama triangular) de las comarcas y provincias de la Comunidad Valenciana, en 1986. 
rural, la Marina Alta y sobre todo el Comtat son precisamente las que, como ya quedó indicado, dan los mayores índices de vejez por término medio.

Valencia presenta una manifiesta dualidad entre un interior bastante envejecido y un litoral con mayor grado de juventud. Efectivamente, todas las comarcas del interior -Canal de Navarrés, Valle de Ayora, Plana de Requena - Utiel, los Serranos y sobre todo el Rincón de Ademuz- dan unas cifras relativas de población vieja superiores al 15\%, e incluso como ocurre en el Rincón de Ademuz, por encima del 30\%. Un espacio más rejuvenecido corresponde al Camp de Túria, Hoya de Buñol, Ribera Alta, Costera, Vall d'Albaida y la Safor. Mientras que son Valencia y su área de influencia extendida por toda l'Horta, los espacios con menor índice de vejez de la provincia, ya que el porcentaje de los de 65 años y más nunca rebasa la cota del $15 \%$.

Finalmente, las comarcas de la provincia de Castellón se caracterizan por índices de vejez elevados en comparación con los de las otras dos provincias. Sólo las Planas Alta y Baixa dan cifras relativas inferiores al 15\% para los mayores de 65 años; ello es debido a la concentración en este ámbito de la mayor parte de los municipios no rurales de esta provincia, incluyendo su capital. L'Alcalatén y el Baix Maestrat, comarcas asimismo litorales y prelitorales, con presencia de importantes centros turísticos y fabriles, tampoco ofrecen niveles excesivamente altos de vejez. Se les puede equiparar al respecto con la Marina Alta y el Comtat, en Alicante, que como ya se señaló, eran las dos comarcas con mayor grado de envejecimiento en esta provincia. Se recalca de esta manera que el grado de envejecimiento es muy superior en la parte Norte de la Comunidad Valenciana.

Todo el interior de la provincia de Castellón está muy envejecido, la población con más de 65 años representa entre el 20 y el 35\% del total, ello sucede en els Ports, Alt Maestrat, Alto Palancia y Alto Mijares. Especialmente intenso es el fenómeno en ésta última, donde la población senil supone casi un tercio del contingente censado.

\section{Grados de envejecimiento por municipios}

Para comprender mejor las repercusiones del fenómeno de envejecimiento sobre los municipios rurales en 1986, se han confeccionado dos mapas según se trate de términos rurales (con menos de 2.000 habitantes) o no rurales (con más de 2.000 habitantes). Destaca el hecho de que entre estos últimos, las tramas son menos oscuras, es decir, tienen un menor grado de vejez. Tan sólo en algunos municipios del interior de la provincia de Valencia, entre los que se encuentran Chelva, Fuente la Higuera y Venta del Moro, La Nucía en la provincia de Alicante, y Cabanes, Vall d'Alba, Villafamés, Villafranca del Cid y Morella, en Castellón, aparece representado el nivel 20-23,9\%. Todos los demás están en situación de «transición» y en muchos casos de «juventud», atendiendo a los criterios establecidos al inicio del presente estudio.

Los municipios rurales, en cambio, casi siempre ofrecen porcentajes de viejos superiores al 20\%. El fenómeno, como ya se ha aludido, se extiende por casi toda la provincia de Castellón, donde se aprecian manchas con la máxima tonalidad en más de una veintena de municipios contiguos. Constituyen el núcleo de determinadas bolsas de vejez que se van difuminando conforme nos acercamos hacia la costa, donde se localizan los núcleos más poblados y con más dinamismo. La mayor parte de los municipios rurales de esta provincia presentan grados de envejecimiento superiores al $24 \%$. La presencia de una economía fuertemente subordinada a la agricultura de secano, combinado con una topografía abrupta, unos suelos de escasa profundidad y unas condiciones térmicas de notable dureza, sobre todo en invierno, condicionan el desarrollo agrícola de este espacio. Los 
cultivos se reducen todavía a la explotación del olivar, frutales de secano y cereales. El aprovechamiento intensivo queda reducido a pequeños espacios de vega, en el fondo de angostos valles, que surcan ríos como el Palancia y Mijares.

Las alternativas a este tipo de agricultura escasamente desarrollada y con nulas perspectivas de mejora a corto plazo, se reducen al aprovechamiento de las masas boscosas -sector en el que se debe afrontar también la contaminación debida a la lluvia ácida-, a la introducción de la ganadería integrada ${ }^{4}$, el turismo de montaña y casos aislados de instalaciones manufactureras: en Villafranca del Cid (Alt Maestrat), industria textil (medias), mueble y de materiales de construcción; talleres de confección e industria alimenticia en Chelva, así como canteras y minas de caolín (que abastecen la fábrica de cemento de Benagéber) en la comarca valenciana de los Serranos; y determinadas descascaradoras de almendra y almazaras, que en muchas ocasiones han cerrado sus puertas por falta de rentabilidad. Estas alternativas en cierta medida han podido detener el éxodo rural, pero no han sido suficiente para revitalizar la demografía de estos municipios. La construcción de centrales hidroeléctricas, como la de Cirat, no revierte en el desarrollo fabril de la región, sino que la energía producida se exporta hacia las grandes áreas urbanas de la plana y litoral de la provincia de Valencia. Tampoco el hecho de haber sido zona de paso entre la Comunidad Valenciana y Aragón ha potenciado la economía de este ámbito. Todavía no están claras las perspectivas de desarrollo derivadas de las actuaciones y programas de ayuda a áreas de montaña regresivas, que han comenzado a ser aplicadas en la Comunidad Valenciana a partir de 1987.

Como ya significó el profesor Gozálvez para 1981, en la provincia de Valencia no existe contigüidad en las bolsas de mayor envejecimiento. Se localizan éstas preferentemente al NW de la provincia y en el Valle de Ayora y la Canal de Navarrés, junto con casos aislados al Sur de dicho ámbito provincial, en pequeños municipios de las comarcas de la Vall d'Albaida y la Safor.

Del mismo modo que vimos en la provincia de Castellón, las tramas son más oscuras entre los municipios rurales que entre los de más de 2.000 habitantes; estos últimos, suelen dar situaciones en la mayoría de los casos de «juventud» (población vieja por debajo del 16\%) y «transición» (población vieja entre el 16 y el 19\%), incluso en los extensos términos del secano interior, Requena, Utiel, Ayora, Buñol, etc.

Los municipios rurales casi siempre presentan índices superiores al 16\%. Sólo algunos términos de la Costera -Estubeny y Genovés-, de la Safor -Almoines-, del Camp del Túria -Marines, Náquera- y de l'Horta -Beniparell-, están por debajo de la indicada cifra relativa. Especialmente desarrollado está el fenómeno de envejecimiento en el Rincón de Ademuz, donde todos los municipios se encuentran en la situación de «muy viejos». Aquí, respecto a 1981, se ha incrementado el número de municipios cuyas cifras de población con más de 65 años rebasan el 28\%. Lo mismo ocurre en determinados espacios municipales de la comarca de los Serranos, como Aras de Alpuente, Alpuente, la Yesa, y también Titaguas. La bolsa de envejecimiento de la comarca de los Serranos se amplía a los términos de Andilla, Chulilla, Calles y Gestalgar; de este modo se comprueba una prolongación sin solución de continuidad del área fuertemente envejecida de la parte SW de la provincia de Castellón.

En el Valle de Ayora y la Canal de Navarrés, como ya se indicó, se centra la segunda gran bolsa de envejecimiento de la provincia de Valencia. Principalmente en los municipios de Jalance, Jarafuel, Teresa de Cofrentes, Bicorp y Quesa; Jarafuel destaca por el hecho de haber acentuado su índice de vejez entre 1981 y 1986, puesto que en la primera fecha no llegaba al $24 \%$, es decir, era un municipio «viejo» (población vieja entre el 20 y el 24 por ciento), pero un quinquenio después, ha pasado a tener un 


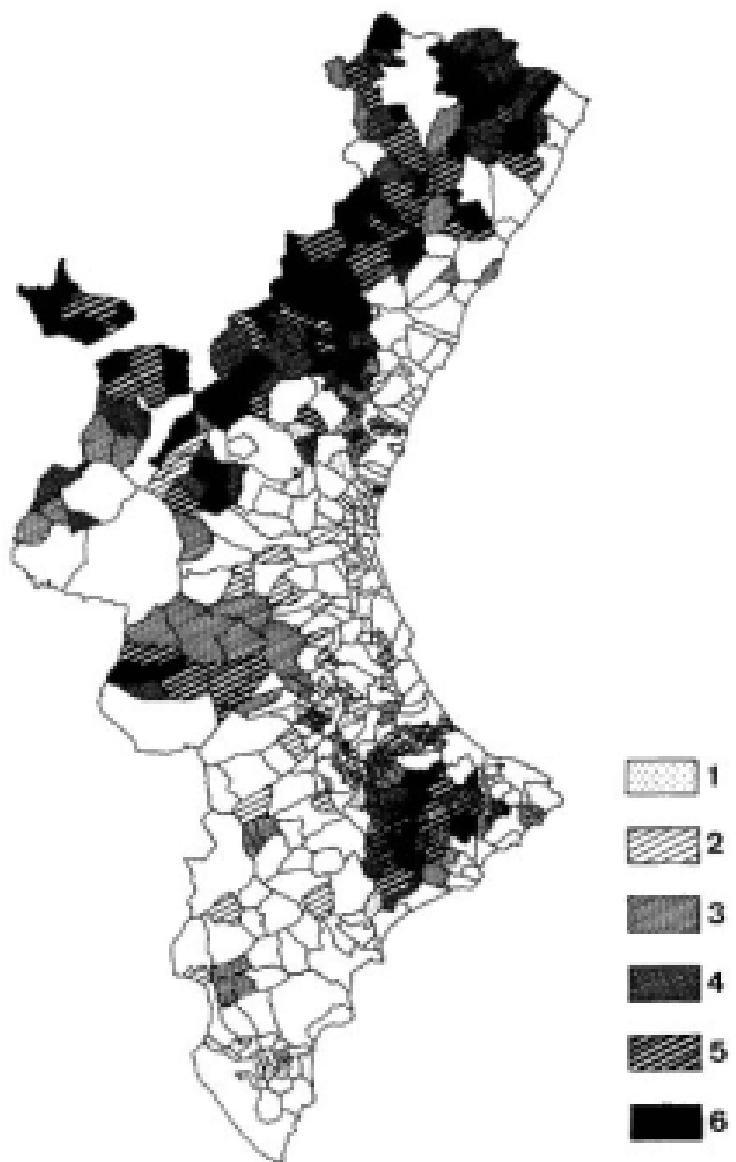

MAPA A: Municipios con menos de 2.000 habitantes de hecho.

$1=$ Menos del $12 \%$.

$2=$ De 12 a $15^{\prime} 9 \%$.

$3=$ De 16 a $19^{\prime} 9 \%$.

$4=$ De 20 a 23 ' $9 \%$.

$5=$ De 24 a $27^{\prime} 9 \%$.

$6=28 \%$ o más. 


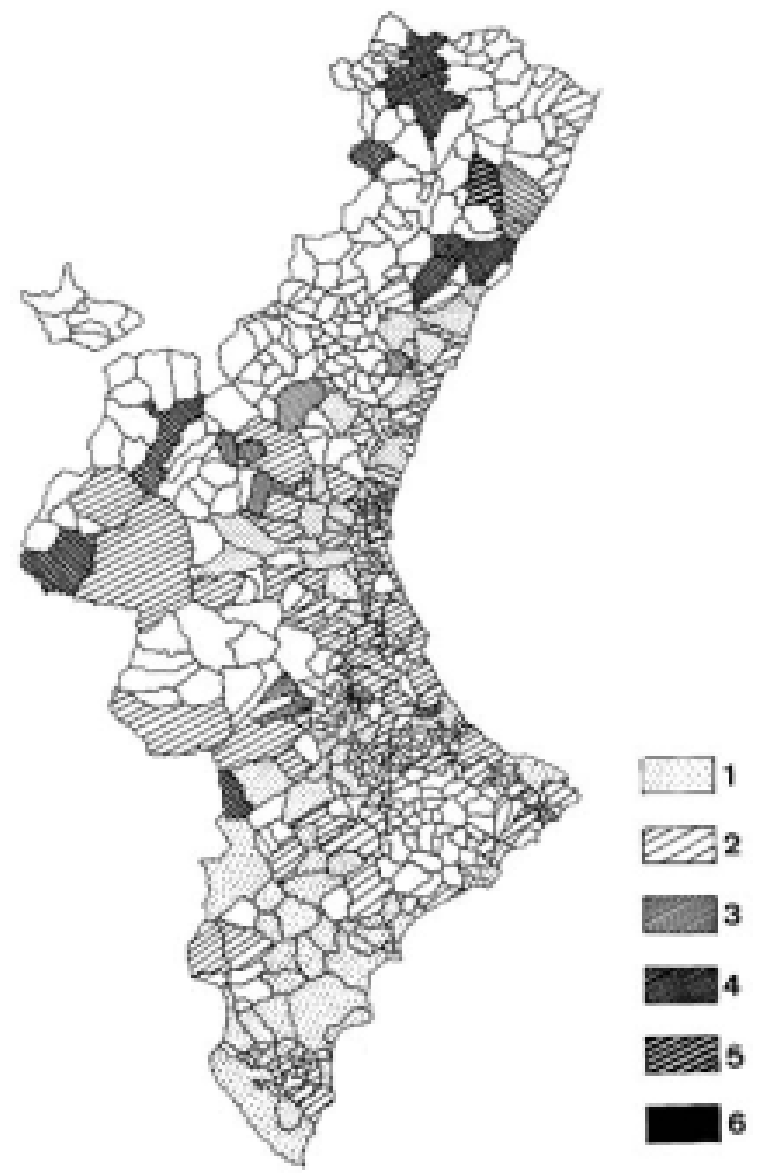

FIGURA 2. Grado de envejecimiento en los municipios de la Comunidad Valenciana (año 1986), según la proporción de los habitantes con 65 años o más.

MAPA B: Municipios con más de 2.000 habitantes de hecho. 
porcentaje de población senil superior al $28 \%$, lo que le convierte en uno de los términos más envejecidos de su provincia.

Los mismos factores ya anunciados para explicar el fuerte grado de envejecimiento del interior de la provincia de Castellón se repiten también en Valencia. Fundamentalmente debe considerarse la dificultad orográfica, con fuertes pendientes de terreno, lo que determina el escaso horizonte de los suelos y sus reducidas posibilidades para el aprovechamiento agrícola. Una red de poblamiento, tradicionalmente muy concentrada, ha permitido del mismo modo el fenómeno del éxodo rural desde los pequeños núcleos, acentuado por la relativa cercanía del área metropolitana de Valencia.

Por último, la provincia de Alicante, muestra una única bolsa de envejecimiento acentuado, que se ubica al $\mathrm{N}$ de la misma, correspondiendo con un área de montaña no excesivamente elevada, pero sí abrupta, e igualmente, con una red tradicional de poblamiento, con altos índices de concentración.

Son las comarcas del Comtat e interior de las dos Marinas, las únicas que tienen municipios con valores superiores al $20 \%$ en lo que respecta a la población con más de 65 años. El núcleo de esta mancha se localiza en la zona de contacto entre el Comtat y las dos Marinas, a través de municipios como Vall de Gallinera, Vall d'Alcalà, Tollos, Facheca y Confrides. A ambos lados de esta línea arbitraria se localizan municipios con altos índices de vejez, aunque, salvo el caso de Bolulla, no rebasan la cifra del $28 \%$.

El resto de la provincia está dominado por la presencia de municipios no rurales con un débil grado de envejecimiento. Entre los de menos de 2.000 habitantes, ninguno tiene más de un $20 \%$ de población por encima de los 65 años. Así, Benitachell (en la Marina Alta), Cañada, Campo de Mirra y Benejama (en el Alto Vinalopó), y Hondón de los Frailes, Hondón de las Nieves (en el Vinalopó Medio) se encuentran en situación de «transición».

Otros términos rurales como Tibi -en la Hoya de Castalla- y todos los del Bajo Segura, dan cifras relativas de envejecimiento muy bajas. La dinámica económica en estos últimos municipios rurales, se ha caracterizado en las últimas décadas, por un desarrollo superior al que se ha producido en la mayor parte de los términos de menos de 2.000 habitantes de la Comunidad Valenciana. El turismo en unos casos, y a partir de él, el fomento del sector construcción, la instalación de diversas plantas manufactureras, y el amplio desarrollo de los regadíos, muchas veces en combinación con espacios de topografía suave, han puesto las bases para retener e incluso atraer elementos de población adulta. De este modo, no se ha acentuado el fenómeno del éxodo rural, y la natalidad se ha mantenido a niveles lo suficientemente altos para atenuar el grado de envejecimiento.

La provincia de Alicante es, por tanto, con la excepción de la bolsa de la Montaña, un espacio bastante rejuvenecido. En cualquier caso, la mayor parte de los municipios rurales se concentran, precisamente, en la citada bolsa de mayor grado de envejecimiento.

\section{Estructura por sexo y edad en los municipios rurales valencianos}

En la figura 3 se representa la estructura por sexo y edad de los municipios de menos de 2.000 habitantes de la Comunidad Valenciana en 1986, distinguiendo dos grandes conjuntos: a) municipios con censo inferior a 1.000 habitantes; b) municipios con censo comprendido entre 1.000 y 1.999 habitantes. Esta distinción nos permite apreciar, dentro de una tendencia global de fuerte grado de envejecimiento, las diferentes estructuras que se dan entre los municipios rurales en función de sus contingentes demográficos.

El perfil de las pirámides correspondientes a los términos con menos de 1.000 habitantes denota que el fenómeno del envejecimiento es aquí mucho más fuerte: la base es muy 
estrecha en comparación con los efectivos de 50 años en adelante. Para toda la Comunidad, el $22,3 \%$ de la población de estos municipios tiene 65 años o más. Si se toma como referencia los 45 años, la proporción asciende al 44\%. En la base, en cambio, sólo el 15'8\% tiene menos de 15 años, y el 22,5\% alcanza los 19 años de edad. Con estos porcentajes, resulta que el 49,0\% de la población entre jóvenes y viejos, depende de unos adultos que apenas suponen poco más de la mitad del censo.

Por provincias, Castellón se caracteriza por ser la de mayor grado de vejez, ya que en los municipios de menos de 1.000 habitantes la población con 65 años o más sube al $26 \%$ del censo, y un $54,4 \%$ para todos los de más de 45 años de edad.

Alicante, en estos niveles demográficos presenta cifras relativas inferiores, el 23 y $51 \%$ respectivamente, y todavía son más bajas las de la provincia de Valencia, con porcentajes de población mayor de 65 años del 19,7\%, y del 45,1\% entre los de más de 45 años de edad.

Según los criterios establecidos, los municipios con menos de 1.000 hab., en 1986, en Castellón son por lo general «muy viejos» (al menos un 24\% de la población tiene 65 años o más), los alicantinos se encuentran en el nivel «viejos» (20 a 23 por ciento), mientras en Valencia dan la situación de «transición» (16 a 19 por ciento).

Las tasas de dependencia están bastantes equilibradas, sin embargo, entre las tres provincias, dado que tanto en Castellón como en Valencia y Alicante un 44-45\% de la población entre jóvenes y viejos depende del 55-56\% de los adultos; hecho que no tiene una explicación homogénea. Así, en Castellón, la cifra de dependientes viene condicionada por el alto índice de mayores de 65 años. En Valencia, por contra, es el contingente de menores de 20 años, que supone el 25\%, lo que determina el volumen de población dependiente. En Alicante, se da la conjunción de ambos hechos.

En estas pirámides dos grupos de cohortes dibujan protuberancias tanto en un sexo como en otro; por un lado, las comprendidas en edades entre 50 y 65 años, y por otro, las cohortes entre 30 y 10 años, aunque ahora no tan claramente definidas.

Se aprecia un déficit importante de población entre los 30 y 50 años, y también, en parte como consecuencia de ello, en la base de la pirámide. Esta ausencia de población adulta obedece a un largo y acentuado proceso de éxodo rural, desarrollado sobre todo durante los años sesenta y setenta. La población joven, en estos momentos, viendo las escasas expectativas de las economías locales de estos pequeños municipios de la Comunidad, decidieron trasladarse hacia centros urbanos, regiones españolas o países con mayor dinamismo económico. Con el paso del tiempo, algunos han regresado a los puntos de origen, contribuyendo a incrementar la proporción de población adulta con mayor edad y de población senil. Otros grupos de jóvenes, en cambio, que tienen entre 20 y 29 años en 1986 (aproximadamente los de 10 a 20 años en los años setenta), por causa de la crisis económica de la pasada década tuvieron mayores dificultades para emigrar, de ahí los contingentes superiores que se aprecian a esta altura de la pirámide respecto a otras cohortes de adultos.

Descenso de la natalidad y emigración no explican, por sí solos, la acentuación del grado de envejecimiento. Ello obedece a la conjunción e intensificación de ambos factores. Primero se produjo la emigración de parejas jóvenes en edad de procrear. Con ello, descendieron las tasas de natalidad. Luego, se produjo un cambio generalizado en el comportamiento reproductor de la población, con un descenso del número de hijos por pareja. Simultáneamente, se incrementó la esperanza de vida y disminuyeron las tasas de mortalidad, con lo que creció aún más la importancia relativa de los viejos.

El hueco que se aprecia entre los grupos de la base de la pirámide se explica por el descenso de los nacimientos ${ }^{5}$, debido en gran medida al referido déficit de población adulta 
en edad de procrear, y al cambio en los comportamientos natalistas -con reducción del número de hijos por mujer casada- experimentado durante las últimas décadas.

Las pirámides referidas a los municipios entre 1.000-1.999 habitantes muestran un menor grado de envejecimiento; si bien, los grupos por encima de 65 años dan un promedio del 17\% para toda la Comunidad, es decir, se encuentran en una situación de «transición», en oposición, a la situación de municipios «viejos» para los de menos de 1.000 habitantes. Aquí, también hay que distinguir entre la provincia de Castellón, que con un 22,6\% está catalogada como provincia «vieja» a estos niveles demográficos; la de Alicante, que es una provincia en «transición» al dar un $16,1 \%$, y la de Valencia, mucho más joven, con menos de un $12 \%$ de población senil.

$\mathrm{Si}$ consideramos que los efectivos por debajo de 20 años representan el 26,7\% del total en toda la Comunidad, el 43,7 \% de población entre viejos y jóvenes dependen de un 56,3\% de población adulta. En Castellón y Alicante, la tasa de dependencia es prácticamente similar a la media de la Comunidad; en Valencia, en cambio, en los municipios entre 1.000 y 1.999 habitantes hay una menor proporción de población dependiente, el 39,9\%.

En definitiva, en estos municipios se aprecia un mayor equilibrio entre todos los grupos respecto a los perfiles de las pirámides de los municipios con menos de 1.000 hab., aunque también se distingue un déficit entre las cohortes correspondientes a edades entre 30 y 50 años, que reflejan asimismo el proceso de éxodo rural en las últimas décadas.

\section{Conclusiones}

Las consecuencias que se derivan de esos porcentajes de ancianos tan altos deben concienciar a la sociedad. Parece que habrá cada vez mayor número de personas mayores que vivirán solas de seguir reduciéndose el número de hijos por familia y también si no se detiene el éxodo rural. Por otro lado, salvo que se produzca un retorno masivo de los emigrados a sus puntos de origen, se acelerará el ritmo de despoblación ${ }^{6}$.

También hay que considerar el conservadurismo entre la mayoría de los individuos de mayor edad, dispuestos más a aceptar las estructuras establecidas que los cambios y los riesgos que comportan las inversiones en reformas agrícolas, concentración parcelaria, constitución de cooperativas, reforma de sistemas de irrigación, etc.

Habrá que plantearse la reordenación de los servicios públicos a nivel local y comarcal. Al descender los volúmenes de población escolar, ya han tenido que cerrar sus puertas las instalaciones docentes de algunos de los pueblos más pequeños. Por contra, con el incremento de la población vieja -sobre todo con el regreso de antiguos emigrantes-, habrá que estudiar detenidamente las dotaciones sanitarias y tratar de encontrar el número óptimo de habitantes por médico para que no se produzca ni déficit ni saturación de facultativos.

Igualmente habrá que calibrar la conveniencia de instalar determinados servicios, planificando las preferencias de los mismos: hogares de pensionistas, campos deportivos, centros culturales y de ocio, parques infantiles; estimando cuantitativa y cualitativamente el nivel de demanda -habitual y de temporada de vacaciones-, deben establecerse esquemas y prioridades.

Actualmente, las tasas de mortalidad parecen crecer ligeramente, consecuencia de la mayor proporción de población anciana. Es la dinámica de la tasa de natalidad, por 

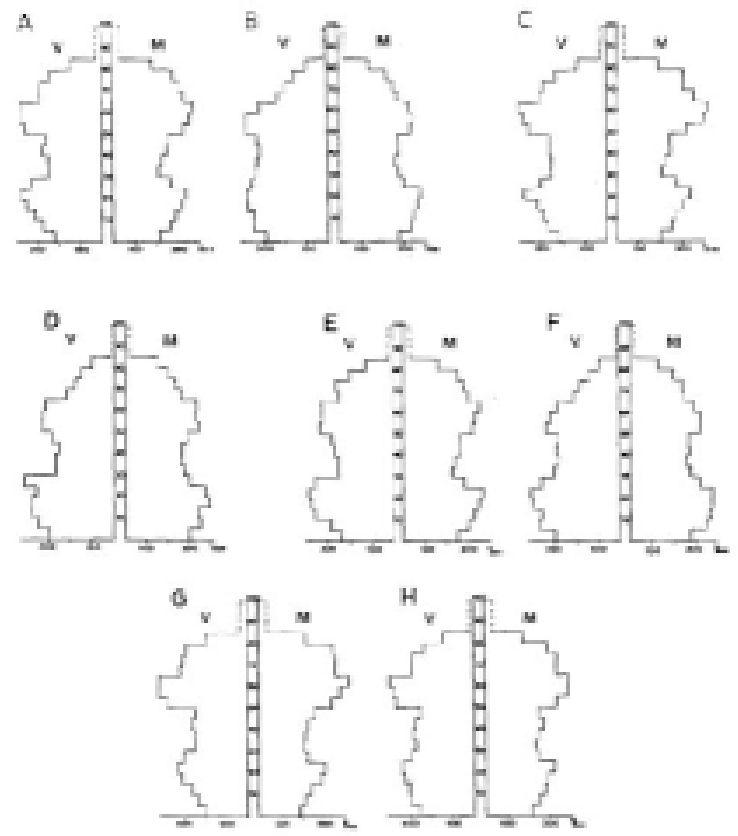

$\mathrm{A}=$ Comunidad Valenciana: Municipios con menos de 1.000 hab.

$\mathrm{B}=$ Comunidad Valenciana: Municipios con más de 1.000 hab.

$\mathrm{C}=$ Provincia de Alicante: Municipios con menos de 1.000 hab.

$\mathrm{D}=$ Provincia de Alicante: Municipios con más de 1.000 hab.

$\mathrm{E}=$ Provincia de Valencia: Municipios con menos de 1.000 hab.

$\mathrm{F}=$ Provincia de Valencia: Municipios con más de 1.000 hab.

$\mathrm{G}=$ Provincia de Castellón: Municipios con menos de 1.000 hab.

$\mathrm{H}=$ Provincia de Castellón: Municipios con más de 1.000 hab.

FIGURA 3. Estructura por edad y sexo en municipios rurales de la Comunidad Valenciana, en 1986. 
tanto, la que va a regular en el futuro el grado de envejecimiento. En este sentido, parece mantenerse su tendencia a la baja, provocando la disminución constante en la cuantía de las nuevas generaciones. Los movimientos emigratorios cada vez tendrán menor incidencia; en todo caso, lo tendrán los retornos.

Posiblemente aumentarán las tasas de dependencia por distintas razones: por el aumento de los efectivos de viejos, debido al envejecimiento de la población local y al regreso de los ausentes que emigraron; por el descenso de la natalidad y la fecundidad; y por la escasa recuperación de los contingentes de población adulta en edad laboral.

Haría falta, por último, poner en práctica medidas basadas en incentivos económicos que modificasen, o al menos frenasen, las tendencias de envejecimiento. Sin embargo, no hay que olvidar, a tenor de los resultados que medidas semejantes han tenido en otros países, que los esfuerzos financieros realizados no se han visto demográficamente correspondidos. De ahí que lo más probable sea el mantenimiento de las tendencias actuales de envejecimiento.

\section{Notas bibliográficas}

1. GOZÁLVEZ PÉREZ, Vicente, «El envejecimiento de los municipios rurales del País Valenciano», Regional Conference on Mediterranean Countries, Zaragoza, 1987.

2. VALLES, Ismael, «Index d'envelliment de la població del País Valencià, segons les dades del Padró Municipal de 1986», en II Jornadas sobre Población Española, Universitat de les Illes Balears, mayo 1989, pp. 425-434.

3. PAILLAT, P., y PARANT, I., Le vieillisement de la campagne française, Paris, INED, Travaux et documents, cahier n. ${ }^{\circ} 88$, 1980, apud GOZÁLVEZ PÉREZ, V., «El envejecimiento de los municipios rurales del País Valenciano», op. cit., p. 317.

4. GOZÁLVEZ PÉREZ, V., «La ganadería integrada en la provincia de Castellón, apoyo a una agricultura familiar en crisis», en Estructuras y regímenes de tenencia de la tierra en España, Ministerio de Agricultura, Madrid, 1987, pp. 267-291.

5. BURRIEL DE ORUETA, E. - CASTELLO TRAVER, J. E., «La transición demográfica en el País Valenciano», en Estudis sobre la Població del País Valencià, (vol. 2), Ed. Alfons el Magnànim e Instituto de Estudios Juan GilAlbert de la Diputación Provincial de Alicante, Valencia, 1988, pp. 618-620.

6. Vid. AA. VV. Espace, Population, Sociétés, 1986, núm. 2, número monográfico dedicado al envejecimiento. Vid. también PAILLAT, P., «El envejecimiento demográfico y sus consecuencias. De la Francia de ayer a la España de mañana», en Estudis sobre la Població..., op. cit., pp. 1125-1133; y la Mesa redonda sobre «Consecuencias socioeconómicas del descenso de la natalidad y envejecimiento de la población», en Ibíd., pp. 1076-1122. 